\title{
A Graph Theoretic Approach for the Identification of Objects Shape Taken from MPEG-7 Database
}

\author{
Jagadeesh Pujari ${ }^{1}$, J.C. Karur ${ }^{2}$, Kumarneeraj Kale ${ }^{3}$ and Kumar Swamy V ${ }^{4}$ \\ ${ }^{1}$ Department of Information Science and Engineering, SDMCET Dharwad, India \\ ${ }^{2 \& 3}$ Department of Computer Science and Engineering, SDMCET Dharwad, India \\ ${ }^{4}$ Department of Electrical and Electronics Engineering, KLEIT, Hubli, India \\ jaggudp@yahoo.com,jck1965@gmail.com,neerajsk066@gmail.com, \\ swamy_bvbdigital@yahoo.co.in
}

\begin{abstract}
Objects never occur in isolation, instead, vary with other objects and in particular environment. In order to recognize the objects efficiently which are similar, there is a need for automating this problem. In this paper, we have proposed an approach to identify objects from MPEG-7 database consisting of 69 classes using graph theory. Graph parameters like graph eccentricity, graph diameter, graph radius and graph center values were used to form the feature vector. Back propagation neural network $(B P N N)$ is used as a classifier. Features were reduced based on their performance in identification. Experimental results prove that an average identification accuracy of $91 \%$ is attained. The study is extended by combining other feature extraction techniques to train the neural network. This work finds its applications to train the robots in automobile industries to handle the objects.
\end{abstract}

\section{Keywords: Object Recognition, Graph features, Shape Classification, Contour}

\section{Introduction}

Image processing refers to a method that converts an image into digital form and performs few operations on it, either to get an enhanced image or to extract useful information from it. Image Processing is among enormously growing technologies today, with its applications in different fields it forms a core research area within computer science and engineering. Object recognition and grouping is also a noteworthy field in Image Processing.

The items are perceived by their shapes, surfaces, hues, forms and numerous different characteristics. Shape is one of the primary low level image features explored in content based image retrieval. Generally there are two types of shape representation methods, namely, the region based and contour based methods. In region-based method, all pixels within a shape region are taken into account to attain the shape representation. Some region-based methods use moment descriptors to describe shape. These include Legendre moments, Zernike moments, geometric moments and pseudo Zernike moments. Since region-based shape representations combine information across an entire object, rather than exploiting information just at the boundary points, they can obtain the interior information of a shape. An advantage of region-based methods is that they can be engaged to describe disjoint and non-connected shapes.

Contour based methods explore only the shape boundary information. There are two types of contour shape modeling: conventional and structural. In the conventional approach, it treats the boundary as a whole, and a feature vector is derived from the boundary detected, which is used to describe the shape. But in the structural approach, it breaks the shape boundary into segments known as primitives, using certain criterion. The 
final representation is usually a string or a tree, and the measure of shape similarity is string matching or graph matching.

Contours consist of curve or edge fragments, which present some meaningful geometric concepts in addition to other features. Contour features can effectively represent objects that can be clearly defined by shape (e.g., a bottle or an LED monitor). Thus, contour features play an important role in object recognition. Therefore, we have considered contour-based shape recognition for the work. Proposed methodology has been divided into four stages. In the first stage we provide images from MPEG-7 database as input, MPEG-7 shape database consists of 1400 silhouette images and contains 70 classes of objects with 20 objects per class. Some sample images of MPEG-7 database are shown in Figure 1. In the second stage we trace the boundary of the shape using bwtraceboundary() function and the contours are extracted using imcontour() function in MATLAB. The third stage involves selecting only the equidistant points from the set of contour points and generating a complete graph using these equidistant points. From the graph we compute the graph parameters such as graph eccentricity, graph diameter, graph radius and graph center. In the final stage, artificial neural network with back propagation learning is adopted to classify the images into several categories which have been taken from MPEG-7 database.
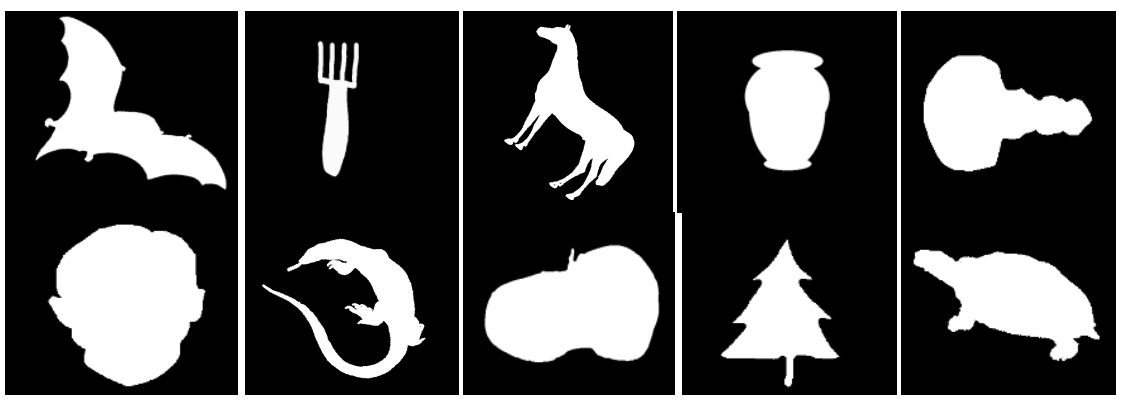

Figure 1. Some Sample Images of MPEG-7 Database

Digital multimedia is a field concerned with computer controlled integration of content, representation, pictures, recordings, sound, and other medium where each sort of data can be spoken to, transmitted and prepared digitally. With the growth of internet, many formats have been proposed for media scenes description. Some of the open standards include VRML1, X3D2, SMIL3, SVG4, MPEG-4 BIFS, MPEG-4, XMT, LaSER, COLLADA5, published by ISO, W3C, etc. Others standards have been proposed by Adobe like Flash or 3ds by Autodesk. The Moving Picture Experts Group (MPEG) is a working group of authorities that was formed by ISO and IEC to set standards for audio and video compression and transmission.

The MPEG standards consist of different Parts. Each part covers a certain aspect of the whole specification. MPEG standards also specify profiles and levels. Profiles are intended to define a set of tools that are available, and levels define the range of appropriate values for the properties associated with them. MPEG-1, 2, 3, 4 and 7 are among the MPEG standards. MPEG-7 is a multimedia content description standard also formally called Multimedia Content Description Interface. While the prior standards focus on coding and representation of audiovisual content, MPEG-7 focuses on description of multimedia content. It addresses content with various modalities including image, video, audio, speech, graphics, and their combinations. MPEG-7 complements the existing MPEG standard suite and aims to be applicable to many existing formats, which include non-MPEG formats and non-compressed formats as well; hence MPEG-7 database was used for the proposed work.

The rest of the paper is organized as follows. Section 2 presents the survey carried out related to the proposed work. It contains 18 research papers from which we have 
considered several ideas. In Section 3, methodology adopted is explained in detail which consists of Image acquisition, Preprocessing, Feature extraction, Classification. The experiments have been performed on MPEG-7 database and results are discussed in Section 4. Finally, Section 5 gives conclusion of the work.

\section{Literature Survey}

In order to know state-of-the-art in contour based recognition, we have carried out a literature survey and following is the gist of the cited papers related to the work carried out.

(Junwei Wang et al., 2012) have done a work on a novel shape descriptor for shape matching and recognizing $2 \mathrm{D}$ object silhouettes by using height function. It gives an astounding discriminative force, which was outlined by fantastic recovery exhibitions on different mainstream shape benchmarks, including MPEG-7 data set, ETH-80 data set and Kimia data set. The proposed descriptor was invariant to geometric changes and harsh to non straight misshapenness. Dynamic programming (DP) algorithm has been used in the matching stage and this technique was compelling under both geometric changes and nonlinear miss happenings.

(Xiang Bai et al., 2007) has proposed an approach on detection and recognition of contour parts based on shape similarity. For each contour part produced by contour grouping, shape similarity is used to retrieve the most similar contour parts in a database of known contour segments. 1-NN classifier yields an overall retrieval rate of 52\% on 10 query shapes from MPEG7 database.

(Haibin Ling and David W. Jacobs, 2005) proposed a methodology for classification of articulated shapes using the inner distance, they showed that the inner distance is articulation insensitive and more effective at capturing complex shapes with part structures than Euclidean distance. They have designed a dynamic programming based method for shape matching and comparison and have been tested on a variety of shape databases including an articulated shape dataset, MPEG7 CE-Shape-1, Kimia silhouettes, a Swedish leaf database and a human motion silhouette dataset. On mpeg-7 database its classification rate reaches $85.40 \%$.

(Boaz J. Super, 2004) proposed a method for fast correspondence-based system for 2D shape classification, they modified the shape retrieval method for use as a shape classifier, and evaluated its accuracy and speed on two test databases. The results are highly encouraging, with low classification error rates and total processing times of less than 1 second. The method produced 96.5\% accuracy for 1400 shapes of MPEG-7 database.

(Boaz J. Super, 2003) proposed a method which improves object recognition accuracy and speed through non-uniform sampling. This article involves methods that improvise the existing methods; this improvisation has been tested on the MPEG-7 database containing 1400 shapes and achieved a classification accuracy of $96.8 \%$.

(Kamal Narseddine et al., 2010) has proposed an approach on multi-scale distance among shapes based upon geodesics in the shape sets. The experimented distance was robust to outliers and uses shape matching to compare shape locally. The resulting similarity measures were found to be invariant to scaling, translation and rotation independently of landmarks or constraints. The proposed approach significantly outperforms previous works and reaches $98.86 \%$ of correct classification rate and $89.05 \%$ of retrieval accuracy.

(Serge Belongie et al., 2002) have presented a method to measure similarity between shapes and produce it for object recognition. In this approach, the measurement of similarity was guided by solving for correspondences among points on the two shapes and using the correspondences to measure an aligning transform. The dissimilarity between the two shapes was estimated as a sum of identical errors among the corresponding 
points. Results were presented for handwritten digits, trademarks, silhouettes and COIL database.

(Manjary P.Gangan et al., 2012) have introduced an automatic image annotation method using MPEG-7 features on fruit images. This method was just a prototype for implementation of automatic image annotation using a fruit image database. The feature vectors were provided for training the KNN-classifier. A comparison of annotation results were made by taking different number of mpeg 7 feature descriptors.

(Manimala Singha et al., 2014) have done a work on content based image retrieval, using features like color and texture known as Wavelet Based Color Histogram Image Retrieval (WBCHIR). The color and texture features were extracted through wavelet transformation and color histogram. The experimental result show that the proposed method outperformed other retrieval methods in terms of average precision and the whole indexing time for the 1000 image database have taken 5-6 minutes.

(Raghuraman Gopalan and Dikpal Reddy, 2008) have proposed a methodology for object classification using shape context. Authors have analyzed the possibility of using 2D shape descriptors to classify different objects present in the scene and examined the effectiveness of three different shape descriptors in capturing various deformations of objects using the standard MPEG7 CEShape-1 dataset.

(Pedro F. Felzenszwalb and Joshua D. Schwartz, 2007) has done a work on hierarchical representation of two dimensional objects that captures shape information at multiple levels of resolution. This representation is based on a hierarchical description of an object's boundary and can be used in an elastic matching framework, both for comparing pairs of objects and for detecting objects in cluttered images. They tested the shape-tree method on the MPEG-7 shape database and obtained a recognition rate of $87.70 \%$.

(Longin Jan Latecki et al., 2000) has proposed an approach for shape descriptors for non-rigid shapes with a single closed contour. The Core Experiment CE-Shape-1 for shape descriptors performed for the MPEG-7 standard gave a unique opportunity to compare various shape descriptors for non-rigid shapes with a single closed contour and achieved a result of $93 \%$.

(Jing Tian et al., 2010) proposed a graph based method for pattern recognition of grid patterns in street network. In this approach, a grid is characterized by a set of mostly parallel lines, which are crossed by a second set of parallel lines with roughly right angle. Experimental result shows the proposed approach is valid in detecting the grid pattern in lower degradation situation.

(Xiang Bai et al., 2009) done a work on integrating contour and skeleton for shape classification. They have illustrated the proposed algorithm on the MPEG-7 dataset, and demonstrated the effectiveness of using the combination of contour and skeleton cues. The results are obtained by averaging a couple of trials. The algorithm achieves $96.6 \%$ classification rate on the MPEG-7 dataset of 70 classes. This suggests that the MPEG-7 dataset might not be sufficient to judge the quality of a shape classification algorithm.

(Yuri Boykov and Vladimir Kolmogorov, 2003) in their work on computing geodesics and minimal surfaces via graph cuts introduced a new segmentation method by combining geodesic active contours and graph cuts. Graph cut algorithms can be used to find globally minimum geodesic contours (minimal surfaces in 3D) under arbitrary Riemannian metric for a given set of boundary conditions. The author mentions this work provides an interesting link between several branches of mathematics-differential geometry, integral geometry, and combinatorial optimization. The main technical problem is solved using Cauchy-Crofton formula from integral geometry.

(Cosmin Grigorescu and Nicolai Petkov, 2003) proposed a method for distance sets for shape filters and shape recognition, they described a two-dimensional (2-D) visual object by the set of (labeled) distance sets associated with the feature points of that object. Based on a dissimilarity measure between (labeled) distance sets and a dissimilarity measure 
between sets of (labeled) distance sets, object segmentation, and shape matching can be addressed. The shape comparison procedure is illustrated on handwritten character classification, COIL-20 database object recognition and MPEG-7 silhouette database retrieval.

(Farzin Mokhtarian and Alan Mackworth, 1986) has done a work on scale-based description and recognition of planar curves and 2D shapes. Path-based Gaussian smoothing techniques are applied to the curve to find zeros of curvature at varying levels of detail. The result is the generalized scale space image of a planar curve which is invariant under rotation, uniform scaling and translation of the curve. The matching algorithm is a modification of the uniform cost algorithm and finds the lowest cost match of contours in the scale space images. It is argued that this is preferable to matching in a so-called stable scale of the curve because no such scale may exist for a given curve.

(Naif Alajlan et al., 2006) has proposed an approach for shape retrieval using trianglearea representation and dynamic space warping. The representation utilizes the areas of the triangles formed by the boundary points to measure the convexity or concavity of each point at different scales. This representation is effective in capturing both local and global characteristics of a shape, invariant to translation, rotation, and scaling, it is robust against noise and moderate amounts of articulation and occlusion. The author has demonstrated the performance of the method using four standard tests on two well-known shape databases.

Table 1. Summary of Literature Survey

\begin{tabular}{|c|c|c|c|}
\hline $\begin{array}{l}\text { Reference } \\
\text { no. }\end{array}$ & Feature & $\begin{array}{c}\text { Classifier/Evaluation } \\
\text { method }\end{array}$ & $\begin{array}{c}\text { Accuracy } \\
(\%)\end{array}$ \\
\hline 1 & $\begin{array}{l}\text { Height function using } \\
\text { contours }\end{array}$ & 1 Nearest neighbor & 90.35 \\
\hline 2 & Contours & 1 Nearest neighbor & 52 \\
\hline 3 & Inner distance & Dynamic Programming & 85.40 \\
\hline 4 & $\begin{array}{l}\text { Contour parts, principal } \\
\text { component analysis }\end{array}$ & Nearest neighbor & 96.5 \\
\hline 5 & $\begin{array}{l}\text { Color structure, edge } \\
\text { histogram, homogenous } \\
\text { texture }\end{array}$ & KNN & NA \\
\hline 6 & $\begin{array}{l}\text { Geometric matching, } \\
\text { IDSC, meta shape context }\end{array}$ & KNN & 92 \\
\hline 7 & Shape contexts & Nearest neighbor & 76 \\
\hline 8 & $\begin{array}{l}\text { Color layout descriptor, } \\
\text { color descriptor, } \\
\text { homogenous texture } \\
\text { descriptor }\end{array}$ & KNN & 82.85 \\
\hline 9 & $\begin{array}{l}\text { Color, texture, shape, } \\
\text { semantic gap of objects }\end{array}$ & MKNN & NA \\
\hline 10 & $\begin{array}{l}\text { Image moments, hue } \\
\text { moments, IDSC }\end{array}$ & SVM & 78 \\
\hline 11 & $\begin{array}{l}\text { Shape boundaries, shape } \\
\text { tree }\end{array}$ & $\begin{array}{l}\text { Shape tree deformation } \\
\text { model }\end{array}$ & NA \\
\hline 12 & $\begin{array}{l}\text { Contour based descriptor, } \\
\text { image based descriptor, } \\
\text { skeleton based descriptor }\end{array}$ & NA & 76 \\
\hline 13 & Graph theoretic operations & Good continuation rule & NA \\
\hline
\end{tabular}




\begin{tabular}{|c|l|l|c|}
\hline 14 & $\begin{array}{l}\text { Contour segment and } \\
\text { skeleton path }\end{array}$ & SVM and boosting & 96.6 \\
\hline 15 & $\begin{array}{l}\text { Geodesic active contours } \\
\text { and graph cuts }\end{array}$ & $\begin{array}{l}\text { Graph cut and Cauchy } \\
\text { Crafton formula }\end{array}$ & NA \\
\hline 16 & $\begin{array}{l}\text { Distance set and labeled } \\
\text { distance set }\end{array}$ & KNN & NA \\
\hline 17 & $\begin{array}{l}\text { Operations on planar } \\
\text { curves }\end{array}$ & Uniform cost algorithm & 95 \\
\hline 18 & Closed contours & Dynamic space warping & \\
\hline
\end{tabular}

From the literature survey, it is observed that Fourier descriptor (FD), curvature scale space descriptor (CSSD), Zernike moment descriptor (ZMD) and texture descriptors are used for shape recognition. The standard databases like MPEG-7, ETH-80 and Kimia-99 are deployed for testing the methods and the summary of it is given in Table 1 . Here the idea of contour converted into graphs and computing graph features from its image is conceived. We are proposing a graph based approach for recognition of objects shape taken from MPEG-7 database.

\section{Methodology}

We have used contour of an object and extracted equidistant contour points. The contour points are joined by lines with a color contrast to the background so as to get a complete graph. The graph features are obtained by treating the complete graph as an object image. Hence, the problem statement is "A graph theoretic approach for the identification of objects shape taken from MPEG-7 image database".

\subsection{Model of the Proposed Work}

The proposed method consists of four stages, In the first stage we provide images from MPEG-7 database as input, MPEG-7 shape database consists of 1400 silhouette images and contains 70 classes of objects with 20 objects per class and are shown in figure 1 . In the second stage we trace the boundary of the shape using bwtraceboundary() function and the contours are extracted using imcontour() function in MATLAB. The third stage involves selecting only the equidistant points from the set of contour points and generating a complete graph using these equidistant points. From the graph we compute the graph parameters such as Graph Eccentricity, graph diameter, graph radius and graph center. In the final stage, artificial neural network with back propagation learning is adopted to classify the images into several categories which have been taken from MPEG-7 database. These stages are shown in figure 2. 


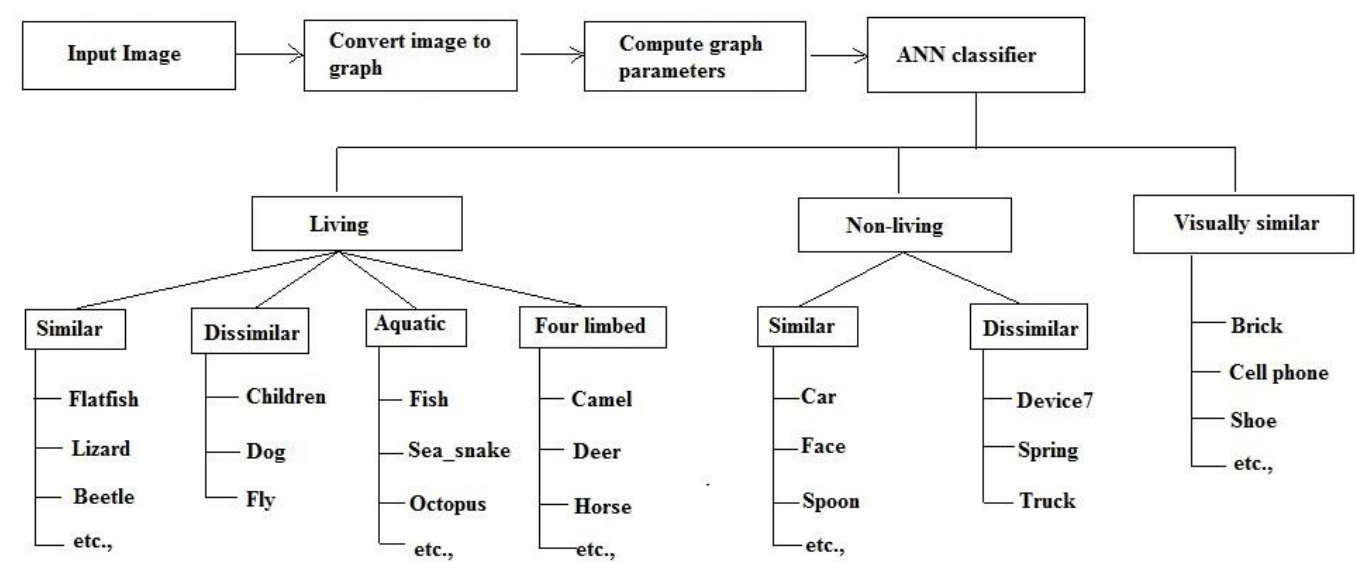

Figure 2. Block Diagram of the Proposed Methodology

\subsubsection{Input Image}

This is the first step of the proposed method. In this step, we have considered images from the MPEG-7 database as input. This database consists of 1400 silhouette images which are grouped into 70 classes with 20 objects per class. In Fig.1, we have shown some images of different categories from MPEG-7 database. In a number of applications, such as document analysis and some industrial machine vision tasks, binary images can be used as the input to algorithms that perform useful tasks.

Gray scale is a range of monochromatic shades from black to white. Therefore, a gray scale image consist only gray shades and no color. While digital images can be treated as gray scale (or white and black) images, even color images consist gray scale data. This is because each pixel has a specific (luminance) value, regardless of its color. Luminance can also be illustrated as intensity or brightness and it can be calculated on a scale from black to white. Most image file formats support a minimum of 8-bit gray scale and it provides 256 levels of luminance per pixel. Few formats support 16-bit gray scale, which provides 65,536 levels of luminance.

\subsubsection{Tracing the Image Boundary}

In this progression, boundary of the picture can be followed to discover whether the limit is constant or not. If the boundary is not in continuity then we need to make it continuous. When we find that limit is nonstop then extract the contours of the picture.

In the proposed strategy we have utilized the bwtraceboundary ( ) function to discover the limit of an image. This function has three parameters like P, bw and fstep. The bwtraceboundary ( ) function seeks the boundary of an object in a binary image bw. $\mathrm{P}$ is a two-component vector indicating the column and segment directions of the point on the object boundary, where you need to start the tracing. What's more, the fstep is a string determining the initial search direction for the following object pixel associated with $\mathrm{P}$ and utilizes strings, for example, ' $\mathrm{N}$ ' for north, 'NE' for upper east, to determine the direction.

The contours of images can be extracted by using many operators like canny, sobel, prewitt etc. In the proposed method we have used the imcontour() function that extracts the contours of images operated on the boundary set bw, and every $100^{\text {th }}$ boundary point is considered in forming the final set of equidistance boundary points to draw the graph. Figure 3 shows the traced boundary points and extracted equidistant points (every 100th) of the input image. 

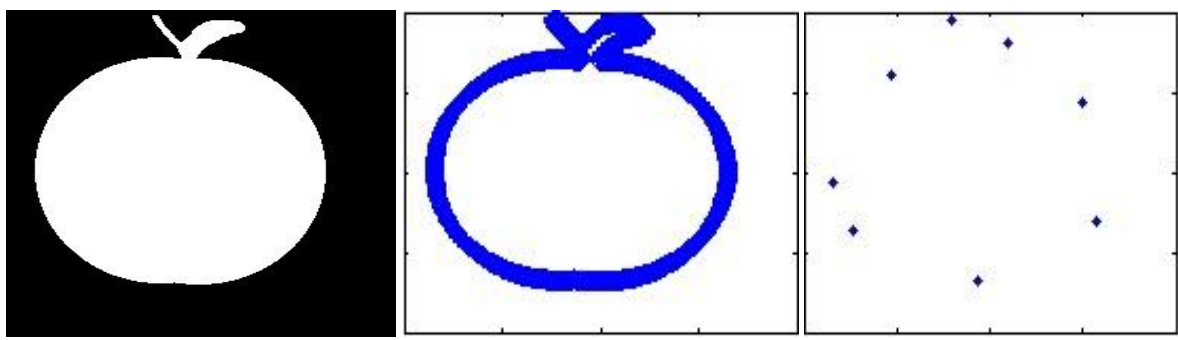

Figure 3. Extracted Equidistant Boundary Points of Apple Image

\subsubsection{Complete Graph}

In the tracing image boundary step we got the contours of the image. By using those contours we have to select the equidistance contour points. In the proposed method we have taken equidistance as 100 and we have also experimented by considering 80,120,150 and 200 as equidistance but we did not get the better performance compared to 100 . Then we plot the complete graph by connecting the equidistance points and finally we will get an acyclic graph of the image and it is shown in Figure 4.

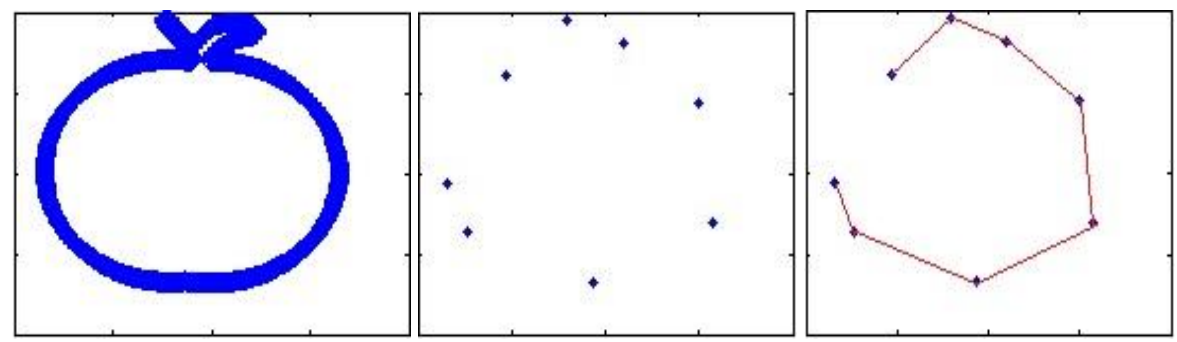

Figure 4. Graph Drawn from the Set of Equidistant Boundary Points

\subsubsection{Computing Graph Parameters:}

Here the graph features are being computed from the complete graph. Graph Features include graph eccentricity, graph diameter, graph radius and graph center. In order to derive these features we first need to calculate Eccentricity of every node belonging to the set of equidistance boundary points. The average values of the graph features computed for different class of image are given in Table 2.

Detailed explanation of graph features being considered is given below:

The eccentricity of a node can be defined as the maximum distance from a node to all other nodes. The distance from a particular node to all other nodes in the graph is considered and among those distances, the eccentricity is the highest of distances calculated.

Let $G$ be a graph and $v$ be a node of $G$. The eccentricity of the node v is the maximum distance from $v$ to any node. That is, $e(v)=\max \{d(v, w): w$ in $V(G)\}$.

The maximum eccentricity value among all the nodes is considered as the eccentricity of the Graph G. The definition of graph diameter follows the definition of graph eccentricity.

I.e., eccentricity $(G)=\max \{e(v): v$ in $V(G)\}$, diameter $(G)=\max \{e(v): v$ in $V(G)\}$

The minimum eccentricity value among all the nodes is considered as the radius of the Graph G.

I.e., Radius $(G)=\min \{e(v): v$ in $V(G)\}$

If the eccentricity of a node is equal to the graph radius, then that node is considered as the center of the graph.

I.e., Center $(G)=\{v$ in $V(G): e(v)=\operatorname{radius}(G)\}$ 
Table 2. Average Values of the Feature Vectors

\begin{tabular}{|c|c|c|c|}
\hline Image class & F1 & F2 & F3 \\
\hline Bat & 8.5 & 4.5 & 5 \\
\hline Beetle & 17.85 & 9.25 & 9.6 \\
\hline Bird & 8.9 & 4.7 & 5.2 \\
\hline Butterfly & 9.65 & 4.9 & 5.75 \\
\hline Camel & 11.05 & 5.7 & 6.35 \\
\hline Cattle & 11.3 & 5.9 & 6.9 \\
\hline Chicken & 10.3 & 5.3 & 5.9 \\
\hline Children & 3.05 & 2 & 2.05 \\
\hline Deer & 17.35 & 9 & 9.35 \\
\hline Dog & 12 & 6.15 & 6.85 \\
\hline
\end{tabular}

\subsubsection{Graph Feature Reduction}

In this step a graph feature is reduced based on its performance. Here we are considering four graph features and we got the values produced by each graph features individually. Graph diameter feature produces same results as that of graph eccentricity therefore we needed to eliminate any one of thsese two features, so we selected the graph diameter feature for elimination. Finally, the four features are reduced to three features in the light of the outcomes they deliver and are given in Table 3.

\section{Table 3. Classification Rate for Graph Features Idividually}

\begin{tabular}{|c|c|c|}
\hline $\begin{array}{c}\text { Feature } \\
\text { Vector }\end{array}$ & Parameter/feature & $\begin{array}{c}\text { Classification } \\
\text { rate (\%) }\end{array}$ \\
\hline F1 & Graph Eccentricity & 53.15 \\
\hline F2 & Graph Diameter & 53.15 \\
\hline F3 & Graph Radius & 52 \\
\hline F4 & Graph Centre & 51 \\
\hline
\end{tabular}

\subsubsection{Classification}

The Artificial Neural Network (ANN) with back propagation learning is used for classification for this work. The different parameters of ANN are used. In the identification process, the output of neural network is represented by a vector of ten values. Each binary value is associated with an animal type. For example, the vector $\left[\begin{array}{ll}1 & 0\end{array}\right.$ $\left.\begin{array}{lllllllll}0 & 0 & 0 & 0 & 0 & 0 & 0 & 0\end{array}\right]$ represent the bat image.

Parameters of ANN are input layers, hidden, output layer and learning constant. In this work, we have 5 number of input neurons for input layer, 5 for hidden layer. TRINGDA is used as transfer function. As there are 10 varieties of classifications, output layer is 10 layers. Learning constant is 0.01 with acceptable error (MSE) as 0.00001 considering 1000 number of epochs. Termination conditions are set based on minimum mean square error (MSE).

\section{Experimental Results}

We have considered 69 classes for experimentation; each class consists of 20 images forming a total of 1380 images. These images are scaled to a definite resolution which is involved in pre-processing stage. Shape boundary of every image is traced and equidistant contour points are extracted from the set of boundary points. Using these contours, graph is drawn from which graph parameters are computed such as graph eccentricity, graph 
radius and graph centre. For experimentation, we have categorized MPEG-7 database classes into living, non-living, aquatic, four-limbed and visually similar. Figure 5 gives the tree structure of the categorization.

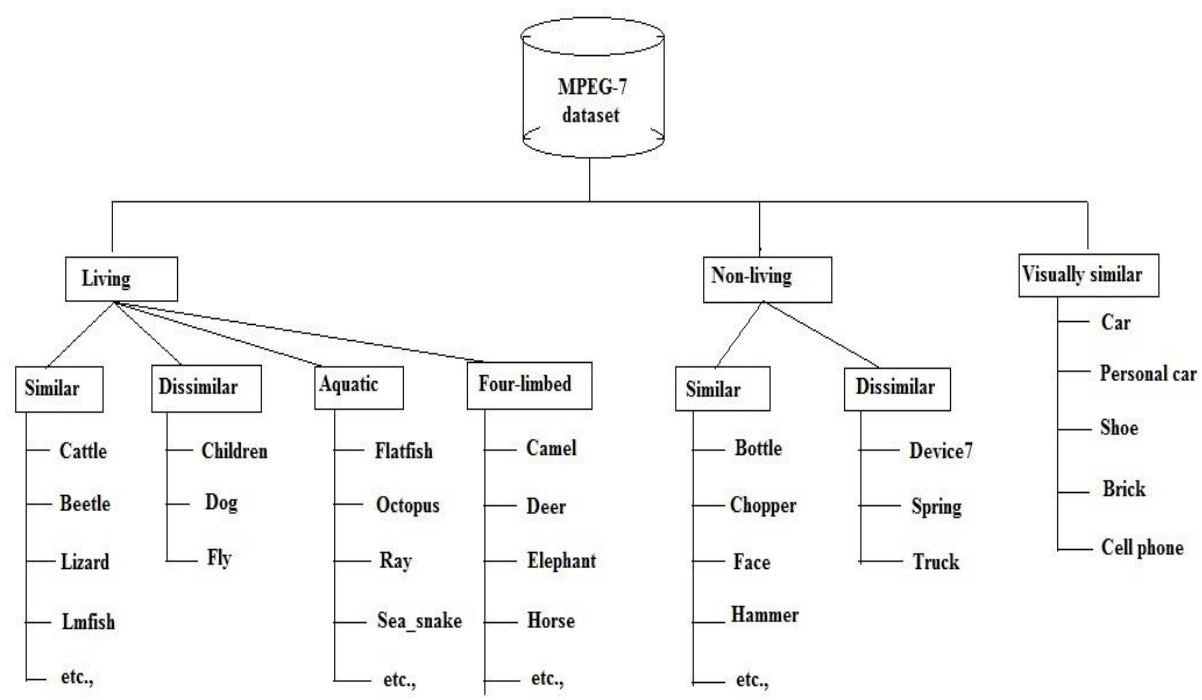

Figure 5. Classification Ttree of the MPEG-7 Dataset

There are three levels of classification, in the first level we are classifying the MPEG-7 dataset into living, non-living and visually similar categories, in the second level we are classifying the living, non-living category into similar, dissimilar, aquatic, and four limbed animals, in the final level of classification we are determining the particular class of the object that is considered.

In the first level of classification, we have randomly selected two classes from living, non-living, and visually similar categories and the graph feature values of these image classes are considered as feature vectors and are trained all at once. The six classes considered include children, rat, fountain, device7, horseshoe, and cell phone, and the classification rate of these classes is $100 \%$, the classification rate is shown in the Figure 6.

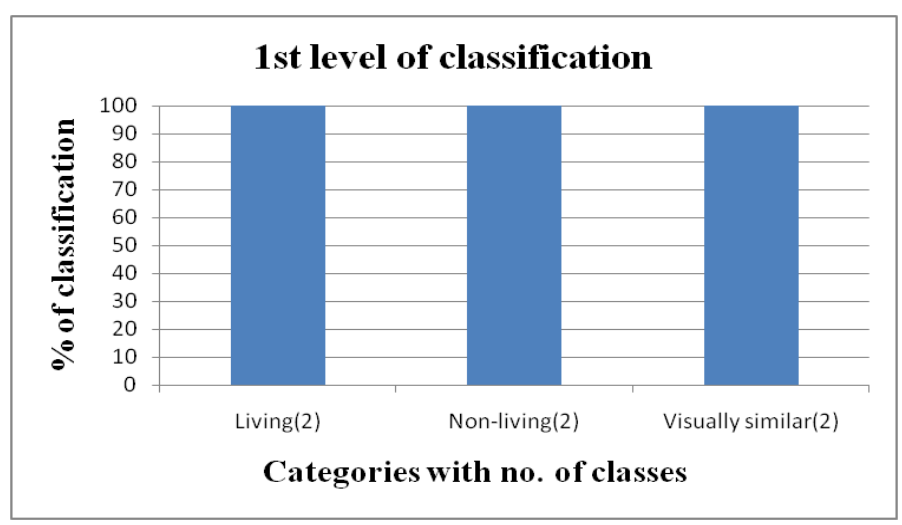

Figure 6. First Level of Classification

\subsection{Living Category}

Here, we have considered animals, birds, and aquatic animals to make living category from the MPEG-7 dataset. We got a total of 24 classes that come under this category out of the 69 classes that are considered for the work, some sample image classes of this 
category are shown in Figure 7. Table 4 gives the average values of the feature vectors considered.

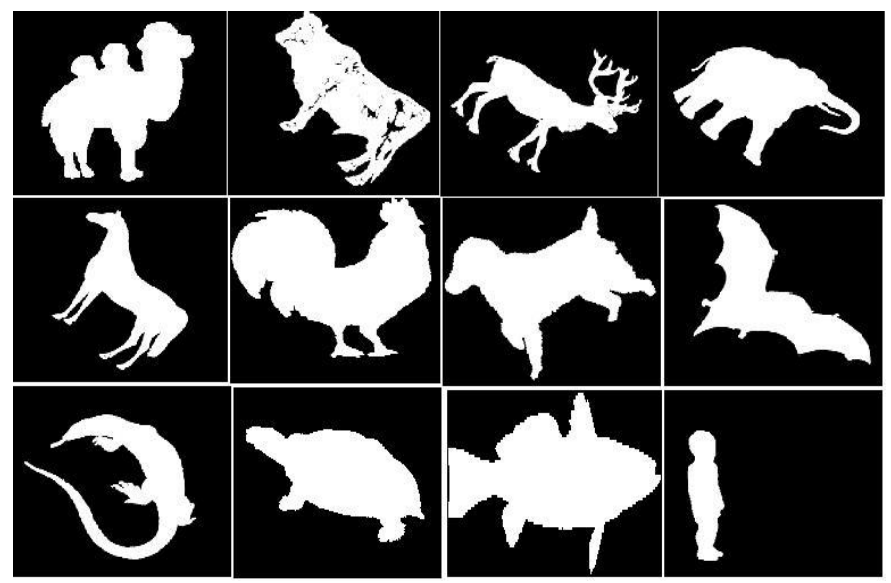

Figure 7. Some Sample Images of Living Category of MPEG-7 Database

Table 4. Average Values of the Feature Vectors of Living Category

\begin{tabular}{|l|c|c|c|}
\hline Image class & F1 & F2 & F3 \\
\hline Bat & 8.5 & 4.5 & 5 \\
\hline Beetle & 17.85 & 9.25 & 9.6 \\
\hline Bird & 8.9 & 4.7 & 5.2 \\
\hline Butterfly & 9.65 & 4.9 & 5.75 \\
\hline Camel & 11.05 & 5.7 & 6.35 \\
\hline Cattle & 11.3 & 5.9 & 6.9 \\
\hline Chicken & 10.3 & 5.3 & 5.9 \\
\hline Children & 3.05 & 2 & 2.05 \\
\hline Deer & 17.35 & 9 & 9.35 \\
\hline Dog & 12 & 6.15 & 6.85 \\
\hline
\end{tabular}

From the above values, feature vectors are created to train the neural network. The classification accuracy of this living category for ten image classes is 55\%, these ten classes considered contained redundant data therefore we eliminated some of the classes and trained them, the classification accuracy increased accordingly. Thus, classification accuracy reached $81.25 \%$ for four image classes and the results are shown in Figure 8 .

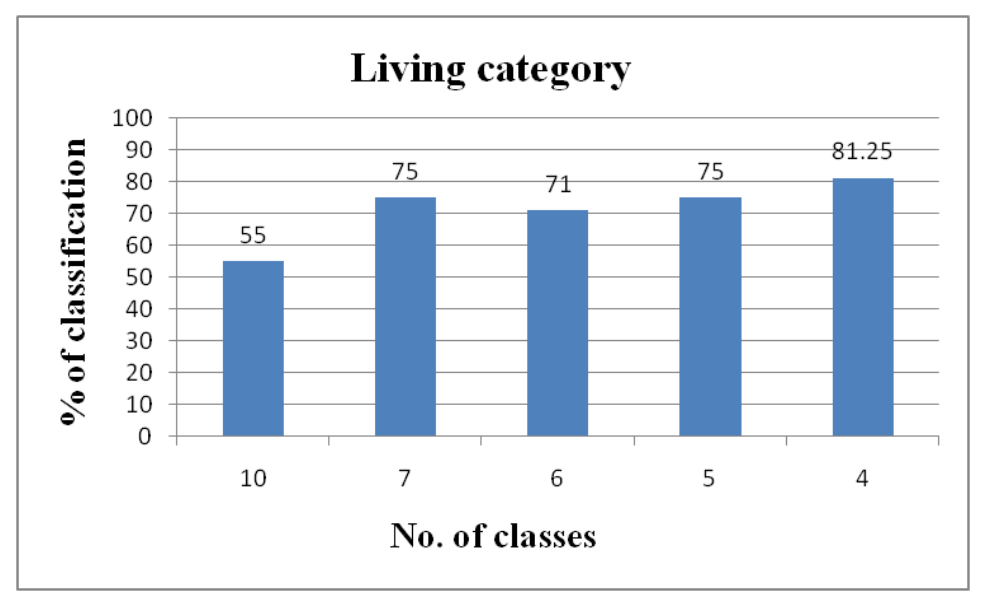

Figure 8. Classification Rate of Living Category 


\subsubsection{Similar and Dissimilar Groups under Living Category}

Here, the living group is further divided into two sub groups namely similar and dissimilar. This sub-division of the living category is based on the values the image classes produce i.e., value of the feature vectors, average values of the feature vectors are given in Table 5 and Table 6 for similar and dissimilar category respectively. The images belonging to these groups are shown in the Figure 9 and Figure 10 respectively.

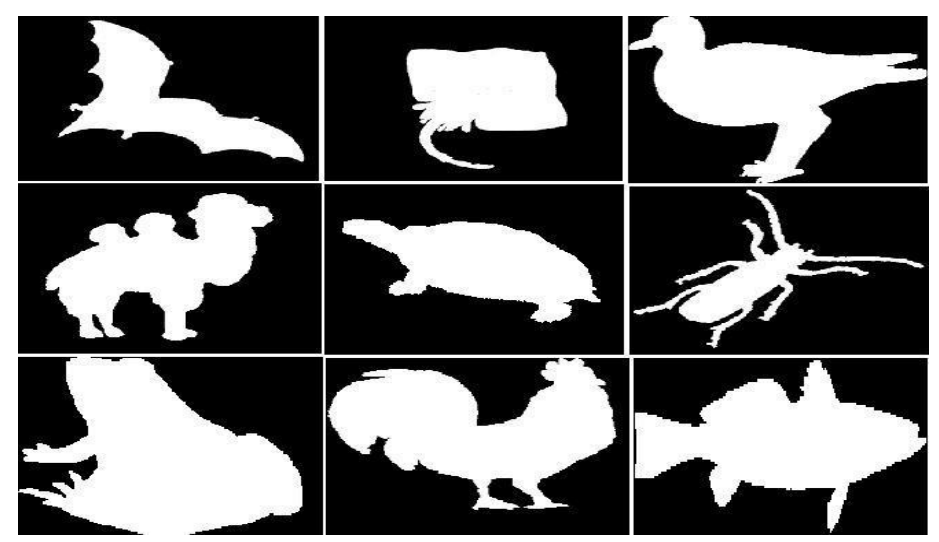

Figure 9. Similar Group Images belonging to Living Category
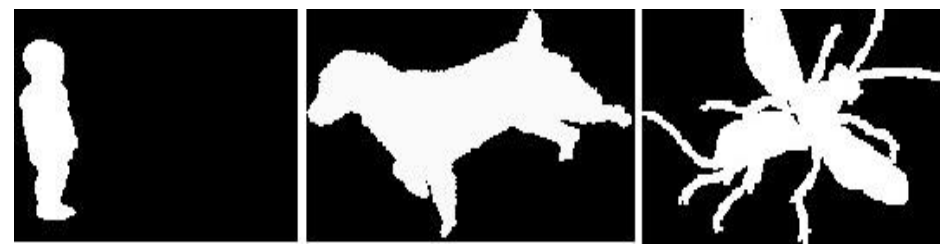

Figure 10. Dissimilar Group Images belonging to Living Category

Table 5. Average Values of the Feature Vectors Considered for Similar Category

\begin{tabular}{|c|c|c|c|}
\hline Image class & F1 & F2 & F3 \\
\hline Beetle & 17.85 & 9.25 & 9.6 \\
\hline Cattle & 11.05 & 5.7 & 6.35 \\
\hline Fish & 10.45 & 5.5 & 5.95 \\
\hline Flatfish & 7.9 & 3.95 & 4.95 \\
\hline Lizard & 14.6 & 7.5 & 8.1 \\
\hline Lumpfish & 8.1 & 4.4 & 4.7 \\
\hline Octopus & 13.65 & 7.05 & 7.6 \\
\hline Sea snake & 9.2 & 4.8 & 5.4 \\
\hline
\end{tabular}

Table 6. Average values of the Feature Vectors Considered for Dissimilar Category

\begin{tabular}{|c|c|c|c|}
\hline Image class & F1 & F2 & F3 \\
\hline Children & 3.05 & 2 & 2.05 \\
\hline Dog & 12 & 6.15 & 6.85 \\
\hline Fly & 20.4 & 10.45 & 10.95 \\
\hline
\end{tabular}


With these three mentioned graph features the classification rate for similar group containing eight image classes of living category is found to be $71.87 \%$ and we also found that the classification rate increased as we decreased the number of image classes for classification as they contained redundant feature values. Thus, we achieved a classification rate of $85 \%$ for five image classes and are pictorially represented in Figure 11. The dissimilar group under living category contained only three classes which include children, fly, and dog and are shown in Figure 10. The classification rate for dissimilar group we attained is $75 \%$ and is shown in Figure 17.

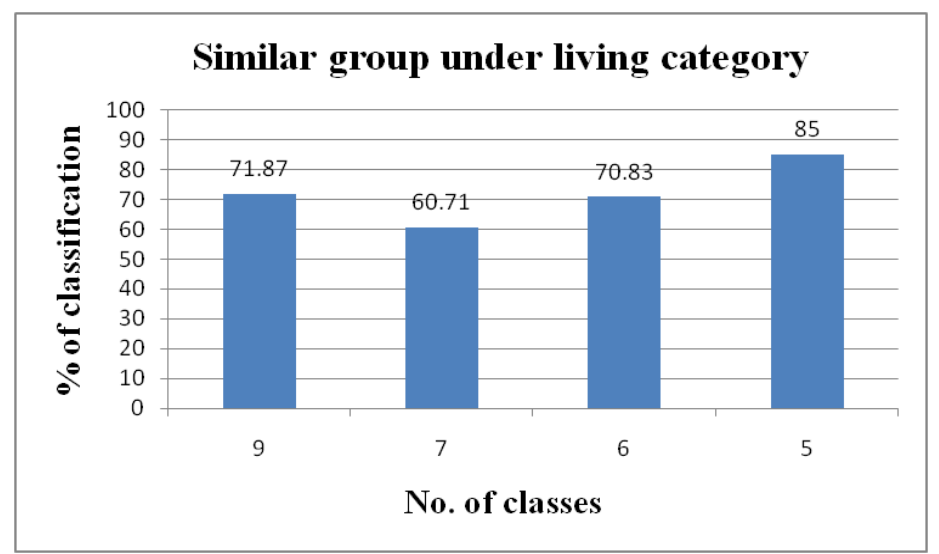

Figure 11. Classification Rate of Similar Group under Living Category

\subsection{Non-living Category}

The non living category of MPEG7 database contained 45 image classes out of the 69 image classes considered for the work; Figure 12 shows some sample image classes belonging to non living category. After processing all the 45 image classes, we considered only 11 image classes for classification, the values of the feature vectors are given in the Table 7.

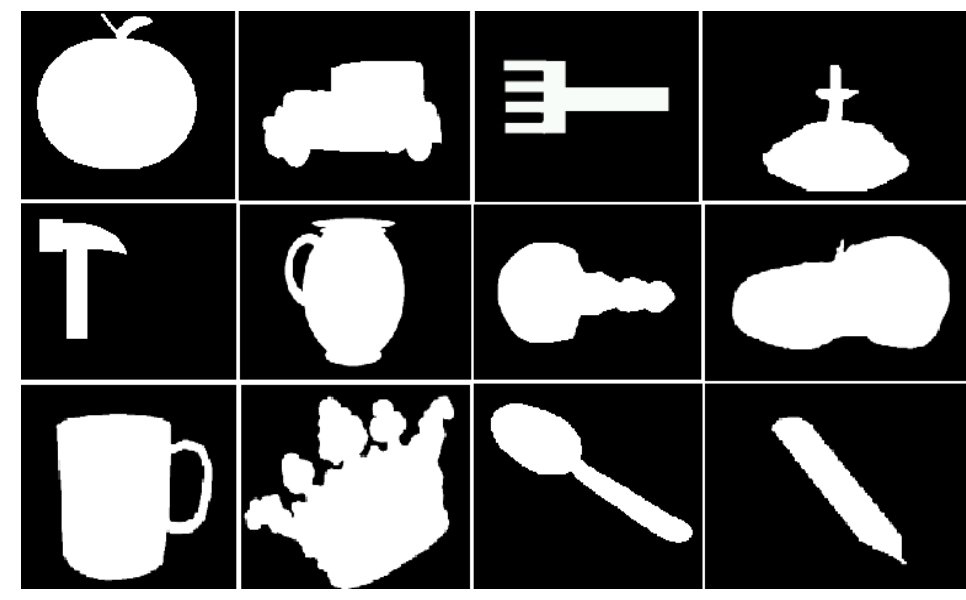

Figure 12. Some Sample Images of Non-living Category 
Table 7. Average Values of the Feature Vectors of Non-living Category

\begin{tabular}{|c|c|c|c|}
\hline Image class & F1 & F2 & F3 \\
\hline Truck & 1.95 & 1 & 1.95 \\
\hline Car & 3.25 & 2 & 2.25 \\
\hline Hammer & 4.7 & 2.65 & 3.05 \\
\hline Cell phone & 5.95 & 3 & 3.95 \\
\hline Fork & 6.9 & 3.65 & 4.25 \\
\hline Chassis & 7.7 & 4.2 & 4.5 \\
\hline
\end{tabular}

Using the values of the feature vectors we found that the classification rate for 11 image classes is $41 \%$. The classification accuracy increased as we descreased the number of classes, as a result we got $64.28 \%, 79.16 \%$, and $90 \%$ for 7, 6, and 5 image classes respectively, the results are shown in the Figure 13.

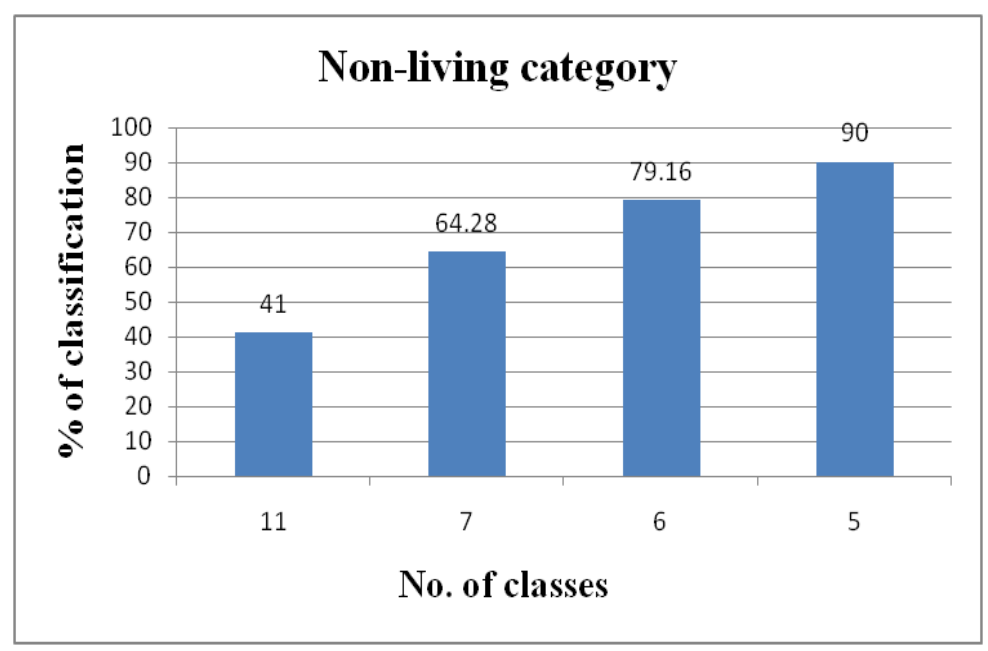

Figure 13. Classification Rate of Non-living Category

\subsubsection{Similar and Dissimilar Groups under Non-living Category}

Out of the 45 image classes of non-living, we got 9 image groups under similar group therefore we chose one image class from each group and hence considered 9 image classes for classification. Some of the image classes belonging to this category are shown in Figure 14. The feature vector values of these image classes are given in the Table 8.

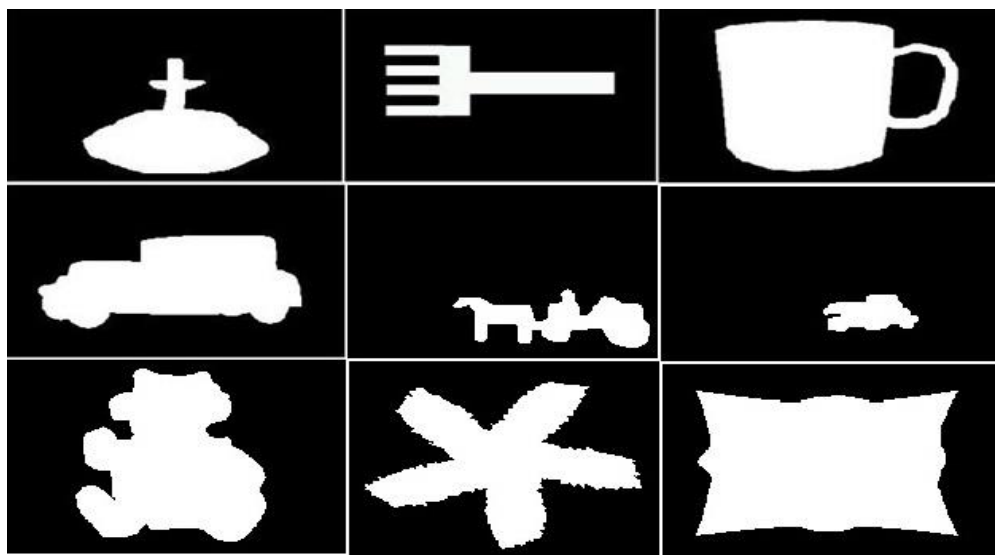

Figure 14. Some Sample Images of Similar Group under Non-living Category 
Table 8. Average Values of the Feature Vectors of Similar Category

\begin{tabular}{|c|c|c|c|}
\hline Image class & F1 & F2 & F3 \\
\hline Stef & 2.85 & 1.8 & 2.05 \\
\hline Car & 3.25 & 2 & 2.25 \\
\hline Hammer & 4.7 & 2.65 & 3.05 \\
\hline Cell phone & 5.95 & 3 & 3.95 \\
\hline Personal car & 6.35 & 3.35 & 4 \\
\hline Classic & 7.7 & 4.2 & 4.5 \\
\hline Bone & 8.15 & 4.2 & 4.95 \\
\hline Device0 & 10.45 & 5.5 & 5.95 \\
\hline Device6 & 11.45 & 6 & 6.45 \\
\hline
\end{tabular}

With these values of the feature vector, the classification rate of these 9 classes found to be $41.6 \%$. Because of the redundant data we reduced the number of classes and got $50 \%, 70.83 \%$, and $85 \%$ for 7,6 and 5 image classes and these results are shown in Figure 15.

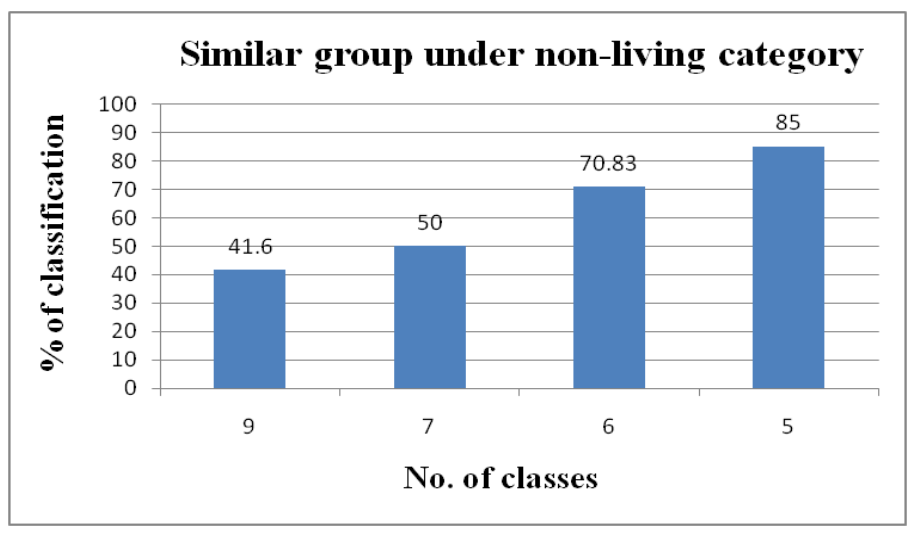

Figure 15. Classification Rate of Similar Group under Non-living Category

There are three image classes that come under dissimilar group of non living category and are shown in Figure 16. The classification rate of the 3 image classes is $66 \%$ and the results are shown in Figure 17.
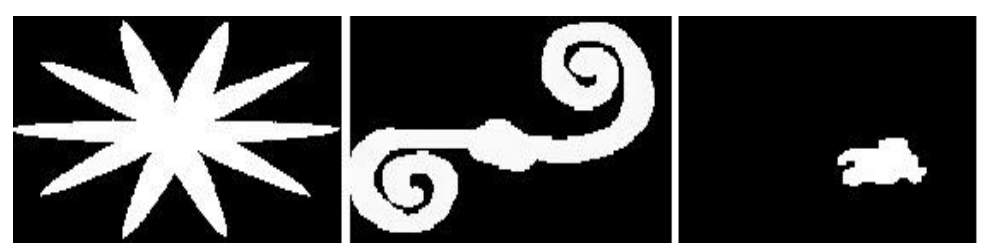

Figure 16. Some Sample Images of Dissimilar Group under Non-living Category 


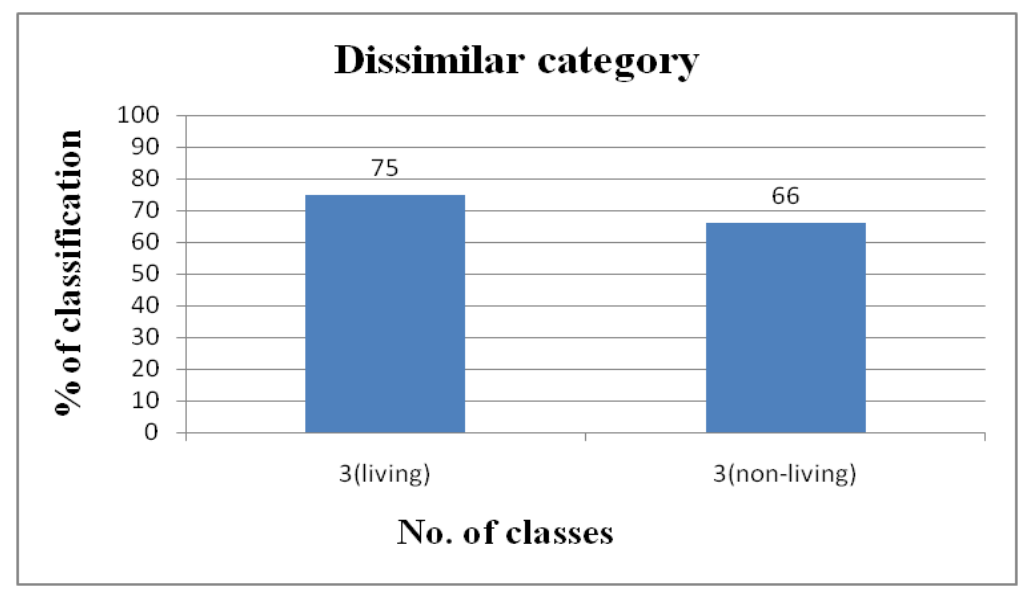

Figure 17. Classification Rate of Dissimilar Group

\subsection{Aquatic Animal's Category}

The aquatic animal's category contained image classes like flatfish, ray, sea snake, fish, lmfish, octopus, frog, and turtle forming a total of 8 classes and some of which are shown in the Figure 18, the values of the feature vectors of these image classes are given in the Table 9. The classification rate of this aquatic category is $75 \%$ for five image classes and the results are shown in Figure 21.

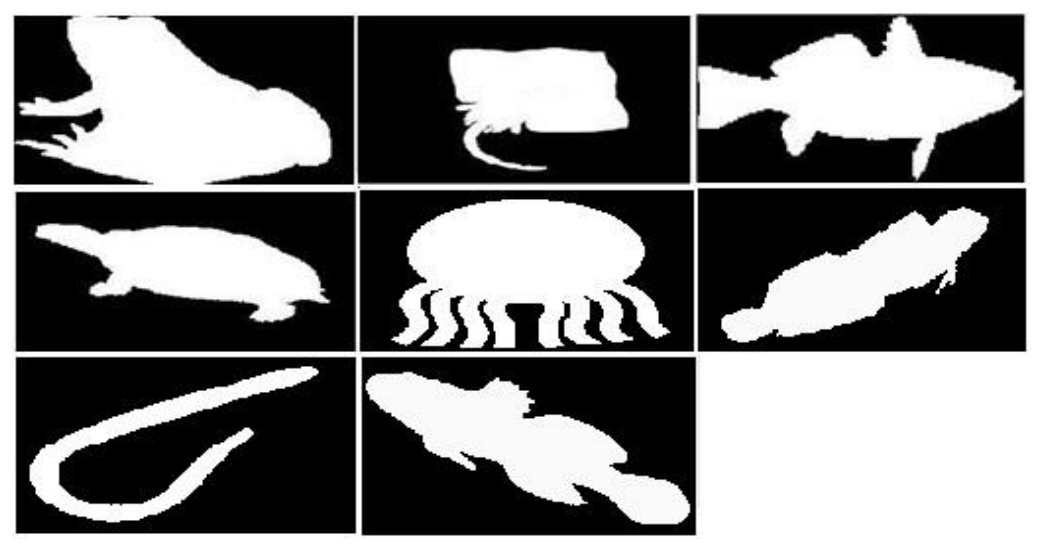

Figure 18. Some Sample Images of Aquatic Animal Category

Table 9. Average Values of the Feature Vectors of Aquatic Animal Category

\begin{tabular}{|c|c|c|c|}
\hline Image class & F1 & F2 & F3 \\
\hline Flatfish & 7.9 & 3.95 & 4.95 \\
\hline Ray & 9.25 & 4.85 & 5.4 \\
\hline Sea snake & 9.2 & 4.8 & 5.4 \\
\hline Fish & 10.45 & 5.5 & 5.95 \\
\hline Lumpfish & 8.1 & 4.4 & 4.2 \\
\hline Octopus & 13.65 & 7.05 & 7.6 \\
\hline Frog & 10.4 & 5.5 & 5.9 \\
\hline Turtle & 8.85 & 4.65 & 5.2 \\
\hline
\end{tabular}

\subsection{Four-limbed Animal's Category}

This category contained image classes like camel, deer, elephant, dog, cattle, rat and horse forming a total of 7 classes and these image classes are shown in the Figure 19, the 
values of the feature vectors of these image classes are given in Table 10. The classification rate of this category is $75 \%$ for six image classes and $70 \%$ for five image classes and the results are pictorially represented in Figure 21.
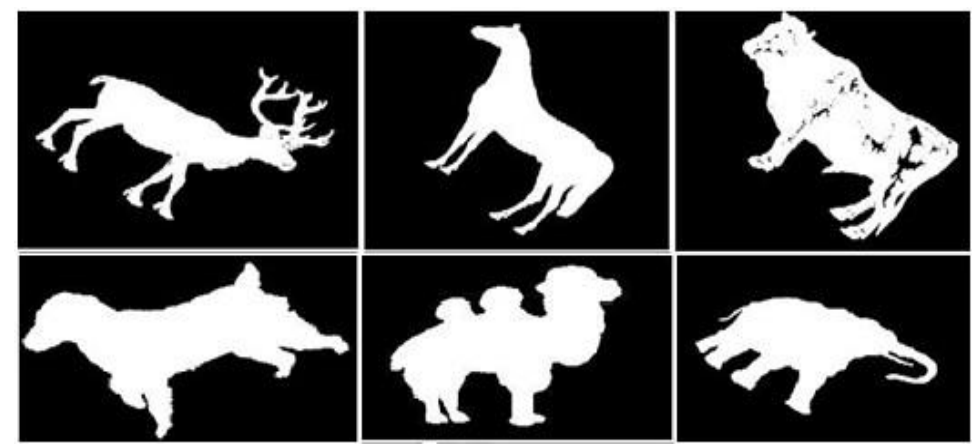

Figure 19. Some Sample Images of Four-limbed Animal Category

Table 10. Average Values of the Feature Vectors of Four-limbed Animal Category

\begin{tabular}{|c|c|c|c|}
\hline Image class & F1 & F2 & F3 \\
\hline Camel & 11.05 & 5.7 & 6.35 \\
\hline Deer & 17.35 & 9 & 9.35 \\
\hline Elephant & 13.45 & 7 & 7.45 \\
\hline Dog & 12 & 6.15 & 6.85 \\
\hline Cattle & 11.3 & 5.9 & 6.4 \\
\hline Rat & 8.1 & 4.1 & 5 \\
\hline horse & 14.95 & 7.6 & 8.35 \\
\hline
\end{tabular}

\subsection{Visually Similar Objects Category}

In this category we are grouping the objects based on their visual similarity, and we got image classes such as shoe, truck, turtle, brick, car, personal car, and cell phones forming a total of seven image classes, these image classes are shown in the Figure 20. The feature vector values are given in Table 11. The classification rate of this category is $75 \%$ for all the seven images classes considered and the results are pictorially represented in Figure 21.

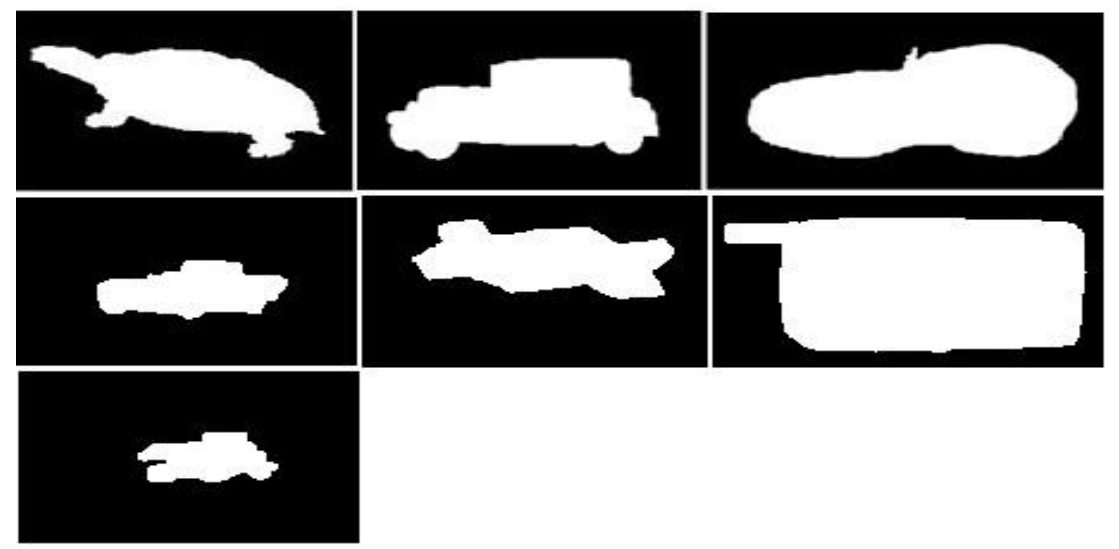

Figure 20. Some Sample Images of Visually Similar Object Category 


\section{Table 11. Average Values of the Feature Vectors of Visually Similar Object Category}

\begin{tabular}{|c|c|c|c|}
\hline Image class & F1 & F2 & F3 \\
\hline Shoe & 6.45 & 3.5 & 3.95 \\
\hline Truck & 1.95 & 1 & 1.95 \\
\hline Turtle & 8.85 & 4.65 & 5.2 \\
\hline Brick & 3.8 & 2 & 2.8 \\
\hline Car & 3.25 & 2 & 2.25 \\
\hline Personal car & 6.35 & 3.35 & 4 \\
\hline Cell phone & 5.95 & 3 & 3.95 \\
\hline
\end{tabular}

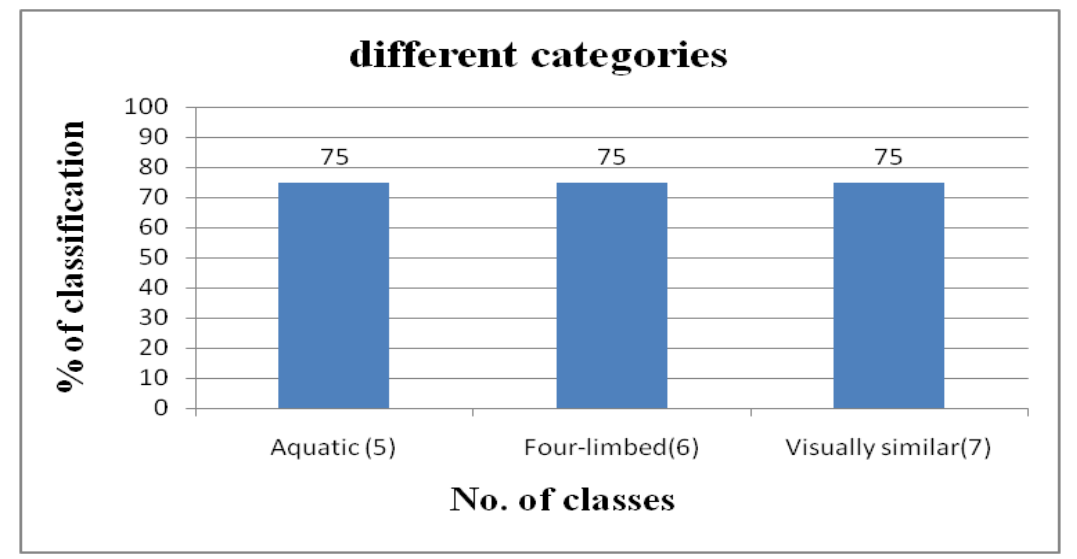

Figure 21. Classification Rate of Various Categories Considered

\section{Conclusion}

The proposed method uses a different approach compared to those exist in the literature. Contour images of MPEG-7 database are converted to a complete graph with certain color. The graph features obtained from the graph image are used to define the shapes of the objects. Only three graph features are used for the 69 image classes of the MPEG-7 dataset in the work, the proposed method has outperformed the state-of-the-art methods and has given an average classification accuracy of $91 \%$ when graph features are used with artificial neural networks.

\section{References}

[1] Junwei Wang, Xiang Bai, Xinge You, Wenyu Liu and Longin Jan Latecki,"Shape matching and classification using height functions", Pattern Recognition Letters 33 (2012) 134-143.

[2] Xiang Bai, Xingwei Yang, Longin Jan Latecki, "Detection and recognition of contour parts based on shape similarity", Pattern Recognition 41 (2008) 2189- 2199

[3] Haibin Ling David W. Jacobs, "Using the Inner-Distance for Classification of Articulated Shapes"

[4] Boaz J. Super, "Fast Correspondence-based System for 2-D Shape Classification"

[5] Amir Egozi, Yosi Keller and Hugo Guterman, "Improving Shape Retrieval by Spectral Matching and Meta Similarity”,IEEE TRANSACTIONS ON IMAGE PROCESSING, VOL. 19, NO. 5, MAY (2010).

[6] Kamal Nasreddine , Abdesslam Benzinou and Ronan Fablet, "Variational shape matching for shape classification and retrieval", Pattern Recognition Letters 31 (2010) 1650-1657.

[7] Serge Belongie, Member, IEEE, Jitendra Malik, Member, IEEE and Jan Puzicha, "Shape Matching and Object Recognition Using Shape Contexts", IEEE TRANSACTIONS ON PATTERN ANALYSIS AND MACHINE INTELLIGENCE, VOL. 24, NO.24, APRIL (2002).

[8] Manjary P.Gangan and Dr. R. Karthi," Automatic Image Annotation by Classification Using Mpeg-7 Features", International Journal of Scientific and Research Publications, Volume 2, Issue 9, September (2012), ISSN 2250-3153. 
[9] Manimala Singha and K.Hemachandran,"Content Based Image Retrieval using Color and Texture", Signal \& Image Processing: An International Journal (SIPIJ) Vol.3, No.1, and February (2012).

[10] Raghuraman Gopalan, Dikpal Reddy, "Object Classification using Shape Context"

[11] Pedro F. Felzenszwalb, Joshua D. Schwartz, "Hierarchical Matching of Deformable Shapes"

[12] Longin Jan Latecki and Rolf Lak"amper and Ulrich Eckhardt, "Shape Descriptors for Non-rigid Shapes with a Single Closed Contour", IEEE Conf. on Computer Vision and Pattern Recognition (CVPR), 424429, (2000).

[13] Jing Tian, Tinghua Ai, Xiaobin Jia, "GRAPH BASED RECOGNITION OF GRID PATTERN IN STREET NETWORKS", the International Archives of the Photogrammetric, Remote Sensing and Spatial Information Sciences, Vol. 38, Part II

[14] Xiang Bai, Wenyu Liu, Zhuowen Tu, "Integrating Contour and Skeleton for Shape Classification"

[15] Yuri Boykov, Vladimir Kolmogorov, "Computing Geodesics and Minimal Surfaces via Graph Cuts", INTERNATIONAL CONFERENCE ON COMPUTER VISION”, NICE, FRANCE, OCTOBER (2003)

[16] Cosmin Grigorescu, Nicolai Petkov, "Distance Sets for Shape Filters and Shape Recognition", IEEE TRANSACTIONS ON IMAGE PROCESSING, VOL. 12, NO. 10, OCTOBER (2003)

[17] Farzin mokhtarian and Alan mackworth, "Scale-Based Description and Recognition of Planar Curves and Two-Dimensional Shapes", IEEE Transactions on Pattern Analysis and Machine Intelligence, VOL. PAMI-8, NO. 1. JANUARY (1986)

[18] Naif Alajlan, Ibrahim El Rube, Mohamed S. Kamel and George Freeman, "Shape Retrieval Using Triangle-Area Representation and Dynamic Space Warping"

\section{Authors}
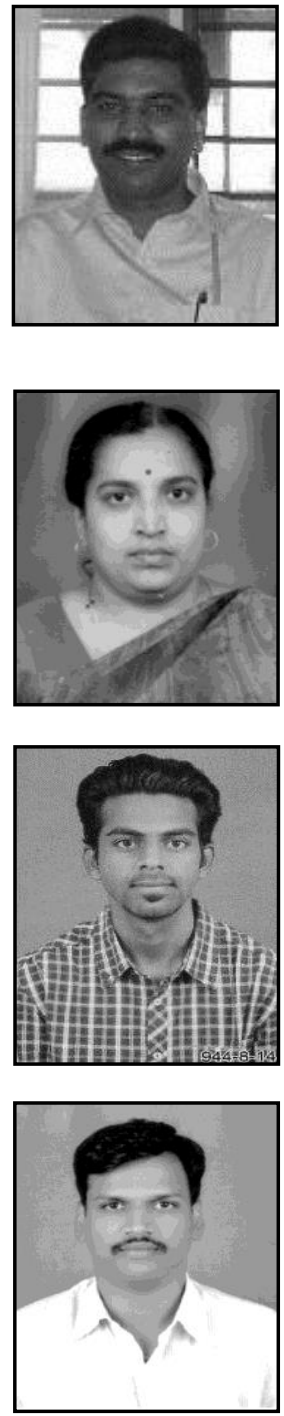

Jagadeesh Pujari has completed his B.E in computer science and engineering, M.S in software systems and $\mathrm{PhD}$ in computer science and engineering. He is currently working as professor and HOD of information science and engineering in SDM college of engineering and technology, Dharwad, Karnataka, India. His research interests are in the field of image processing and pattern recognition. $\mathrm{He}$ is a reviewer for international journals and conferences.

J.C. Karur received B.E degree in Electronics and communication from B.V.B college of engineering and technology in Hubli in 1986, M.Tech degree in computer science and engineering, SJC Mysore in 1992. She is currently a professor within SDMCET Dharwad and pursuing her $\mathrm{PhD}$.

Kumarneeraj Kale received B.E degree in computer science and engineering from B.V.B college of engineering and technology, Hubli in the year 2014. Pursuing M.Tech in Computer science and engineering from SDMCET, Dharwad.

V. Kumar Swamy is presently working as Assistant Professor in Department of Electrical and Electronics Engineering, K.L.E. Institute of Technology, Hubli. He completed his B.E (Electrical and Electronics) in 2006 and M.Tech (Digital Electronics) in 2009. $\mathrm{He}$ is pursuing his $\mathrm{PhD}$ from VTU, Belagavi. He has total of 8 years of experience. 
International Journal of Database Theory and Application Vol.10, No.3 (2017) 